\title{
ST Reha: Richtungswechsel in der Tarifstrukturentwicklung
}

\section{Beatrix Meyer}

Leiterin Abteilung Stationäre Versorgung und Tarife, FMH

\author{
Der Verwaltungsrat der SwissDRG AG hat beschlossen, die Einführung von ST Reha \\ zu verschieben. Dies, damit direkt eine neue Tarifstruktur basierend auf Kodes der \\ Prozedurenklassifikation CHOP entwickelt und per 1. Januar 2022 eingeführt wer- \\ den kann. Die FMH hat gemeinsam mit den Fachgesellschaften bereits Vorschläge \\ zur Abbildung rehabilitativer Leistungen in der CHOP erarbeitet.
}

Die fertiggestellte Tarifversion ST Reha 0.5 wird definitiv nicht eingeführt. Das hat der Verwaltungsrat der SwissDRG AG am 1. Juni 2018 entschieden. Die FMH bedauert diesen Entscheid nach der jahrelangen Entwicklungsarbeit. Die Krankenversicherer und Kantone hatten allerdings das Modell des indirekten Leistungsbezugs von ST Reha 0.5 bemängelt. Deshalb wird die SwissDRG AG eine neue Tarifstruktur mit einem direkten Leistungsbezug erarbeiten. Dazu sollen die von den Rehabilitationskliniken erbrachten Leistungen anhand von Kodes der Prozedurenklassifikation CHOP erfasst und in die Tarifstruktur ST Reha integriert werden. Abgesehen davon wird die künftige Tarifstruktur eine Entscheidungsbaumlogik aufweisen. Die Einführung des neuen Tarifmodells ist per 1. Januar 2022 vorgesehen.

Abbildung 1: Basisleistung in der Rehabilitation

(basierend auf den Vorschlägen von FMH und Fachgesellschaften)

1. Mindestmerkmale, die für alle Reha-Arten gleich definiert sind:

Eintrittsabklärung

Behandlungsplan

Angaben zu Therapie und Schulung, z.B. Dauer einerTherapieeinheit

Visite mit Facharzt

Rehabilitations-Koordination oder -Teambesprechung

Austrittsplanung

2. Mindestmerkmale, die für jede Reha-Art spezifisch definiert sind:

- Ergänzende, fachspezifische Parameter zur Eintrittsabklärung

Zusätzliche Visiten, ausser der Facharztvisite

- Definition derTherapie und Schulung, z.B. welcheTherapie ist in welchem Umfang obligatorisch zu erbringen; welche verschiedenenTherapieformen können patientenbezogen kombiniert werden; wie ist die Mindesttherapiedichte pro Woche definiert

- Zusätzliche Austrittsplanung

- Austrittsassessments zusätzlich zur Erhebung des ADL-Scores (Activities of Daily Living)

Eine detaillierte Beschreibung dieser Mindestmerkmale für die Basisleistungen in der Rehabilitation finden Sie unter www.fmh.ch $\rightarrow$ Stationäre Tarife $\rightarrow$ Rehabilitation ST Reha.

\section{Vorhersehbarer Richtungswechsel}

Für die FMH kommt der Richtungswechsel nicht überraschend. Der Verwaltungsrat der SwissDRG AG hatte nämlich bereits 2016 einen mittelfristigen Wechsel auf eine stärker leistungsorientierte Tarifstruktur durch

Die Abbildung von Basis- und Zusatzleistungen in der CHOP bildet die Grundlage für die neue ST Reha-Version.

die Integration von CHOP-Kodes ins Auge gefasst. Allerdings war die Rehabilitation in der CHOP ungenügend abgebildet. Die FMH hat deshalb noch im selben Jahr ihre Arbeiten zur Definition von Leistungen in der Rehabilitation aufgenommen. Ziel war es, eine Lösung zu finden, damit nur so viele neue CHOP-Kodes wie nötig geschaffen werden müssen. Dies, um den administrativen Aufwand für die Leistungserfassung so gering wie möglich zu halten.

\section{FMH und Fachgesellschaften definieren Basisleistungen}

Für die Ärzteschaft ist es zentral, dass die Qualität in der Rehabilitation gewährleistet ist. Es muss deshalb festgelegt werden, welche Leistungen ein Patient in der stationären Rehabilitation mindestens erhalten sollte. Gemeinsam mit den Fachgesellschaften hat die FMH deshalb für jede Reha-Art ${ }^{1}$ Vorschläge für die Definition der Basisleistung erarbeitet. Die Basisleistung einer Reha-Art wurde dabei anhand von Mindestmerkmalen festgelegt. $\mathrm{Zu}$ unterscheiden sind einerseits Mindestmerkmale, die für alle Reha-Arten gleich ausgestaltet sind. Beispielsweise soll immer spätestens 
2 Die FMH hatte sich für eine Lösung ohne CHOP-Kodierung der Basisleistung eingesetzt. Eine Abbildung der Basisleistungen durch Umgestaltung der Hauptkostenstellen in der medizinischen Statistik der Krankenhäuser wäre dabei eine Lösungsmög lichkeit gewesen. Das BFS ist diesem Vorschlag de FMH jedoch nicht gefolgt.

Korrespondenz:

FMH

Baslerstrasse 47

CH-4600 Olten

Tel. 031359111

Fax 0313591112

tarife.spital[at]fmh.ch drei Tage nach Eintritt der Patientinnen und Patienten ein Behandlungsplan vorliegen. Andererseits sind je nach Reha-Art weitere spezifische Mindestmerkmale vorgesehen (vgl. Abb. 1). Ergänzend dazu sind auch Minimalqualifikationen und -vorgaben zu definieren, die zur Durchführung einer Leistung erfüllt bzw. gegeben sein müssen. Beispielsweise gilt es festzulegen, welche Qualifikation die Leitung eines Behandlungsteams vorweisen muss oder welche Therapeuten es im Behandlungsteam braucht.

\section{Zusatzleistungen in der Rehabilitation}

Zusätzlich zu diesen Basisleistungen erbringen Rehabilitationskliniken je nach Spezialisierung und Patientenstruktur bei Bedarf auch verschiedene Zusatzleistungen. Beispielsweise können indikationsbezogene Pflegeleistungen wie eine engmaschige Überwachung der Vitalparameter nach einem Schädel-HirnTrauma oder eine aufwendige Wundbehandlung erforderlich sein. Die FMH hat gemeinsam mit den Fachgesellschaften bereits solche Zusatzleistungen definiert. Schon 2017 haben die Fachgesellschaften entsprechende Anträge für CHOP-Kodes beim Bundesamt für Statistik (BFS) eingereicht. Zusätzlich haben die Fachgesellschaften mit Unterstützung der FMH be-

\section{Abbildung 2: Zusatzleistungen in der Rehabilitation}

(basierend auf den Anträgen von FMH und Fachgesellschaften)

1. Neuer CHOP-Kode für den indikationsbezogenen Zusatzaufwand

Der indikationsbezogene Zusatzaufwand wird anhand von definierten Punkten pro Leistung und pro Tag erfasst. Pro Tag ist eine maximale Punktzahl festgelegt. Die Gesamtzahl der Punkte pro Aufenthalt ergibt einen CHOP-Kode.

Für folgende Bereiche sind Zusatzleistungen definiert, die mit CHOP 2019 erfasst werden können:

- Fachübergreifende, indikationsbezogene Pflege

- Erkrankungen des Herz-Kreislauf-Systems, z.B. nach Klappenoperation

- Erkrankungen der Atmungsorgane, z.B. bei Pulmonaler Hypertonie

- Erkrankungen des zentralen Nervensystems, z.B. Anwendung von Robotik

- Verletzungen oder postoperative Zustände, z.B. nach Amputation der unteren Extremität

Aufwendige Wundbehandlung

Psychische Funktionseinschränkungen

Intensive Beratungsgespräche

Eine detaillierte Beschreibung dieser Zusatzleistungen in der Rehabilitation finden Sie unter www.fmh.ch $\rightarrow$ Stationäre Tarife $\rightarrow$ Rehabilitation ST Reha

\section{Anpassung bestehender CHOP-Kodes}

Folgende bestehende CHOP-Kodes wurden für die CHOP 2019 an die Gegebenheiten der Rehabilitation angepasst:

- Isolierung (Erweiterung der Anzahl Behandlungstage)

- Diagnostischer Hausbesuch (neu auch Anwendung für die Rehabilitation)

- Berufsrehabilitation (Präzisierung des bestehenden Kodes)

- Behandlung von Atemregulationsstörungen ausserhalb IPS/IMC (Überarbeiten eines bestehenden Kodes)

antragt, dass vier bestehende CHOP-Kodes auf die Bedürfnisse der Rehabilitation angepasst werden (vgl. umgesetzte Anträge in Abb. 2).

\section{Definitionen fliessen in die CHOP 2019}

Das BFS und seine Expertengruppe, in welcher die SwissDRG AG, die Versicherer und Kantone sowie H+ und die FMH vertreten sind, haben die Vorschläge der FMH und ihrer Fachgesellschaften zur Definition von Basis- und Zusatzleistungen mehrfach geprüft und diskutiert. Anregungen der Partner wurden aufgegriffen und nach Möglichkeit integriert. Inzwischen hat das BFS die Definitionen für die Basis- und Zusatzleistungen der Rehabilitation in die nächste per 1. Januar 2019 gültige CHOP-Version aufgenommen. Wichtig ist, dass die CHOP-Kodes von den Kliniken erfasst werden, damit die SwissDRG AG ihr neues ST-Reha-Modell anhand

Wichtig ist, dass die per 1. Januar 2019 gültigen neuen CHOP-Kodes von den Kliniken erfasst werden.

valider Daten kalkulieren kann. Für jeden stationären Reha-Fall muss dabei nur ein CHOP-Kode für die Basisleistung kodiert werden. ${ }^{2}$ Dazu kommen bei Bedarf die CHOP-Kodes für allfällige Zusatzleistungen. Die sorgfältige Erfassung der Leistungen ist auch deshalb zentral, weil sie es erlaubt, rasch zu erkennen, ob für künftige CHOP-Versionen Anpassungen oder Ergänzungen notwendig sind. Entsprechende Vorschläge können beim BFS im jährlichen Antragsverfahren eingereicht werden.

\section{Weitere Arbeiten zeitnah vorantreiben}

Mit den vorliegenden Definitionen der Basis- und Zusatzleistungen in der Rehabilitation ist eine wichtige Grundlage für die neu zu entwickelnde kodegestützte Tarifstruktur ST Reha geschaffen. In einem nächsten Schritt wird es nun darum gehen, dass die SwissDRG AG gemeinsam mit den Partnern ein Konzept für die Entwicklung der neuen Tarifstruktur erstellt. Im Weiteren müssen für die Vergütung von teuren Leistungen Lösungen erarbeitet werden, wobei auch nicht Reha-spezifische Leistungen wie z.B. Dialysen oder teure Medikamente zu berücksichtigen sind. Auch eine klare Abgrenzung von ST Reha zu den anderen Tarifstrukturen ist erforderlich. Für die Einführung von ST Reha bis am 1. Januar 2022 bleibt also noch viel zu tun. 